Vol. 46 (1992) [381-384]

\title{
ON STRONGLY RIGHT BOUNDED FINITE RINGS II
}

\author{
WEIMIN XUE
}

\begin{abstract}
An associative ring $R$ is called a $B T$-ring if $R$ is strongly right bounded, but not right duo, and not strongly left bounded. We show that the order of the smallest $B T$-rings (without unity) is 16 , while we prove earlier that the order of the smallest unitary $B T$-rings is 32 .
\end{abstract}

From [3], an associative ring $R$ is right (left) duo if every right (left) ideal is an ideal, and $R$ is strongly right (left) bounded if every nonzero right (left) ideal contains a nonzero ideal. The interesting result [3, Proposition 6] states that a ring is right duo if and only if every factor ring is strongly right bounded. For convenience, we call $R$ a $B T$-ring in case $R$ is strongly right bounded, but not right duo, and not strongly left bounded.

Let $p$ be a prime number and $\mathbb{Z}_{p}$ the field with $p$ elements. In view of Birkenmeier $\left[1\right.$, Example 9] we note that the unitary ring $\left[\begin{array}{cc}\mathbb{Z}_{p} & \mathbb{Z}_{p} \\ 0 & \mathbb{Z}\end{array}\right]$ is a $B T$-ring. Birkenmeier and Tucci [3, Example 8] constructed a finite unitary $B T$-ring with 32 elements, and recently the author [5] proved that 32 is just the order of the smallest unitary $B T$ rings. In this paper, we shall consider finite $B T$-rings (not necessarily possessing a unity) and show that the smallest such rings have 16 elements. Related questions are also discussed.

If $R$ is a ring and $r \in R$, we let $\langle r\rangle_{R}\left(R\langle r\rangle, R\langle r\rangle_{R}\right)$ denote the right (left, twosided) ideal of $R$ generated by $r$. For a finite ring $R$, let $|R|$ denote the order of $R$ and $\operatorname{char}(R)$ denote the characteristic of $R$.

Using an idea of [3, Example 8], we first give a $B T$-ring with 16 elements.

EXAMPLE 1: Let $\left\langle x^{3}\right\rangle$ be the ideal of the polynomial ring $\mathbb{Z}_{2}[x]$ generated by $x^{3}$. Let $S=\mathbb{Z}_{2}[x] /\left\langle x^{3}\right\rangle$ and $R=\left[\begin{array}{ll}0 & S \bar{x} \\ 0 & S \bar{x}\end{array}\right]$. Let $a=\left[\begin{array}{ll}0 & \bar{x} \\ 0 & 0\end{array}\right]$ and $b=\left[\begin{array}{ll}0 & 0 \\ 0 & \bar{x}\end{array}\right]$. The right ideal $\langle b\rangle_{R}$ is not an ideal since $a b \notin\langle b\rangle_{R}$. So $R$ is not right duo. The minimal right ideals $\langle a b\rangle_{R},\left\langle b^{2}\right\rangle_{R}$ and $\left\langle a b+b^{2}\right\rangle_{R}$ are ideals, so $R$ is strongly right bounded. Since

Received 12 November 1991

This research is supported by the National Science Foundation of China

Copyright Clearance Centre, Inc. Serial-fee code: 0004-9729/92 $\$ A 2.00+0.00$. 
$(a+a b) b=a b \notin{ }_{R}\langle a+a b\rangle$, the minimal left ideal ${ }_{R}\langle a+a b\rangle$ is not an ideal. So $R$ is not strongly left bounded. It follows that $R$ is a $B T$-ring with 16 elements.

We need the following propositions to prove that the ring $R$ in the above example is one of the smallest $B T$-rings.

Proposition 2. [4] A finite ring $R$ is commutative if the order $|R|$ of $R$ has square free factorisation.

Proposition 3. Let $R$ be a ring with $p^{2}$ elements. If $R$ is strongly right bounded then it is right duo.

ProOF: Each non-zero proper right ideal $I$ of $R$ has $p$ elements. Hence $I$ is a minimal right ideal that is two-sided.

Recall that there are exactly two (up to isomorphism) noncommutative rings with order $p^{2}$; one is $S_{1}=\left[\begin{array}{cc}\mathbb{Z}_{p} & \mathbb{Z}_{p} \\ 0 & 0\end{array}\right]$ and the other one is $S_{2}=\left[\begin{array}{ll}\mathbb{Z}_{p} & 0 \\ \mathbb{Z}_{p} & 0\end{array}\right]$, where $S_{1}$ is right duo but not strongly left bounded and $S_{2}$ is left duo but not strongly right bounded. In [5] we proved that a unitary ring with $p^{4}$ elements is strongly right bounded if and only if it is strongly left bounded. For rings with $p^{3}$ elements we have:

PROPOSITION 4 . Let $R$ be a ring with $p^{3}$ elements. If $R$ is strongly right bounded but not right duo, then $R$ is strongly left bounded, that is, any ring with order $p^{3}$ is not a $B T$-ring.

Proof: Assume we have a $B T$-ring $R$ with $p^{3}$ elements.

Since $R$ is strongly right bounded but not right duo, there is a principal right ideal $B$ which is not an ideal and $B$ contains a non-zero ideal $I$. Then $|B|=p^{2}$ and $|I|=p$. Now $R$ is not strongly left bounded, so there is a minimal left ideal $L$ that is not an ideal. We see that $R / I$ is not strongly right bounded, so we may assume $R / I=\left[\begin{array}{ll}\mathbb{Z}_{p} & 0 \\ \mathbb{Z}_{p} & 0\end{array}\right]$, where $(L+I) / I=\left[\begin{array}{cc}0 & 0 \\ \mathbb{Z}_{p} & 0\end{array}\right]$ and $B / I=\left[\begin{array}{cc}\mathbb{Z}_{p} & 0 \\ 0 & 0\end{array}\right]$. Then

$$
p=|(L+I) / I|=|L /(L \cap I)|=|L|,
$$

where $L \cap I=0$ since $L$ is a minimal left ideal but not an ideal. Therefore $L={ }_{R}\langle c\rangle=$ $\{j c \mid 0 \leqslant j<p\}$ where $c+I=\left[\begin{array}{ll}0 & 0 \\ 1 & 0\end{array}\right]$. And let $B=\langle b\rangle_{R}$ where $b+I=\left[\begin{array}{ll}1 & 0 \\ 0 & 0\end{array}\right]$. We have that $R=L+B$. Since $R$ is not commutative, $\operatorname{char}(R)=p$ or $p^{2}$.

(1) If $\operatorname{char}(R)=p^{2}$ then $I={ }_{R}\langle p b\rangle_{R}$. We have $c b=c+n p b$, where $n p b \neq 0$ since $L={ }_{R}\langle c\rangle$ is not a right ideal. Let $b^{2}=b+m p b$, where $0 \leqslant m<p$. Then $c b^{2}=c b+n p b^{2}$, that is, $c(b+m p b)=c b+n p(b+m p b)$. Since $c p=0$ and $p^{2} b=0$ we see that $0=n p b$, a contradiction. 
(2) If $\operatorname{char}(R)=p, R$ is a $\mathbb{Z}_{p}$-algebra. The Dorroh extension of $R$ via $\mathbb{Z}_{p}$, denoted by $\left(R ; \mathbb{Z}_{p}\right)$, is the standard one with the operations on $R \times \mathbb{Z}_{p}$ of

(i) $\left(r_{1}, a_{1}\right)+\left(r_{2}, a_{2}\right)=\left(r_{1}+r_{2}, a_{1}+a_{2}\right)$,

(ii) $\left(r_{1}, a_{1}\right)\left(r_{2}, a_{2}\right)=\left(r_{1} r_{2}+r_{1} a_{2}+a_{1} r_{2}, a_{2} a_{2}\right)$,

yielding $\left(R ; \mathbb{Z}_{p}\right)$ is a unitary ring with $p^{4}$ elements. Since $R$ is not strongly left bounded, neither is $\left(R ; \mathbb{Z}_{p}\right)$. By [5, Theorem], $\left(R ; \mathbb{Z}_{p}\right)$ is not strongly right bounded, either. Now $R$ is strongly right bounded, so it follows from [2, Corollary 1.5] that there exists $(r, a) \in\left(R ; \mathbb{Z}_{p}\right)$ with $a \neq 0$ such that $r t+a t=0$ for all $t \in R$. Then $e t=t$ where $e=-a^{-1} r$, that is, $e$ is a left identity of $R$. If $e \in B, c=e c \in B$, a contradiction since $L \cap B=0$. So $e=j c+b^{\prime}$ for some $b^{\prime} \in B$ and $0<j<p$, and then $b=e b=\left(j c+b^{\prime}\right) b=j c b+b^{\prime} b$. Then $j c b=b-b^{\prime} b \in B$ and so $c b \in B$. It follows that $B$ is a left ideal of $R$, a contradiction.

Now we are ready to prove our main result.

THEOREM 5. The order of the smallest BT-rings is 16 .

Proof: The $B T$-ring in Example 1 has 16 elements. Let $R$ be a finite ring and $|R|<16$. We shall prove that $R$ is not a $B T$-ring. Since $|R|<16$, we have the following two cases to be considered:

Case (1): $|R|=p^{n}$ for some prime number $p$; then we must have $n \in\{1,2,3\}$ since $|R|<16$. Then by Propositions 2,3 , and $4, R$ cannot be a $B T$-ring.

Case (2): $|R|=q^{m} p^{n}$ where $q$ and $p$ are two distinct primes. It follows that either $m=1$ or $n=1$, since $|R|<16$. Without loss of generality, we assume that $|R|=q p^{n}$ where $n \in\{1,2\}$. Since any finite ring $R$ is a direct sum of rings of prime power order, we let $R=R_{1} \oplus R_{2}$ where $\left|R_{1}\right|=q$ and $\left|R_{2}\right|=p^{n}$. From Case (1) we see that $R_{2}$ is not a $B T$-ring. Now $R_{1}$ is commutative by Proposition 2 ; hence $R=R_{1} \oplus R_{2}$ is not a $B T$-ring.

If $R$ is a finite unitary $B T$-ring, then, according to [5], $|R|$ must have a factor of the form $p^{5}$. One may ask, if $R$ is a finite $B T$-ring without unity, does $|R|$ have a factor of the form $p^{4}$ ? The following example gives a negative answer.

EXAMPLE 6: Let $\mathbb{Z}_{2}[x] /\left\langle x^{2}\right\rangle=\{0,1, x, 1+x\}$. Let $X=\{0, x\}$ and $A=\{0,1\}$. Consider the ring $R_{1}=\left[\begin{array}{ll}0 & X \\ X & A\end{array}\right]$. The right ideal $\left[\begin{array}{ll}0 & 0 \\ X & A\end{array}\right]$ is not an ideal. So $R_{1}$ is not right duo. The minimal right ideals $\left[\begin{array}{ll}0 & 0 \\ X & 0\end{array}\right]$ and $\left[\begin{array}{ll}0 & X \\ 0 & 0\end{array}\right]$ are ideals, so $R_{1}$ is strongly right bounded. Similarly, $R_{1}$ is also strongly left bounded but not left duo. One notes that $|R|=8$. Let $R_{2}=\left[\begin{array}{cc}\mathbb{Z}_{3} & \mathbb{Z}_{3} \\ 0 & 0\end{array}\right]$, which is right duo but not strongly left bounded. Now $R=R_{1} \oplus R_{2}$ is a $B T$-ring with $2^{3} \cdot 3^{2}$ elements. 
In the above example, neither $R_{1}$ nor $R_{2}$ is a $B T$-ring but $R=R_{1} \oplus R_{2}$ is a $B T$-ring. However, if $R=R_{1} \oplus R_{2}$ is a finite unitary $B T$-ring, then at least one of $R_{1}$ and $R_{2}$ must be a $B T$-ring. This follows from [5, Proposition 3] which states that a finite unitary ring is right duo if and only if it is left duo.

We conclude this paper with the following questions: Does there exist a (unitary) $B T$-ring with 16 (32) elements that is not isomorphic to the $B T$-ring $R$ of Example 1 [3, Example 8]?

\section{REFERENCES}

[1] G.F. Birkenmeier, 'Split-null extensions of strongly right bounded rings', Publ. Mat. 33 (1989), 37-44.

[2] G.F. Birkenmeier and H.E. Heatherly, 'Embeddings of strongly right bounded rings and algebras', Comm. Algebra 17 (1989), 573-586.

[3] G.F. Birkenmeier and R.P. Tucci, 'Homomorphic images and the singular ideal of a strongly right bounded ring', Comm. Algebra 16 (1988), 1099-1112, 2661-2662.

[4] B.D. Erickson, 'Orders for finite noncommutative rings', Amer. Math. Monthly 73 (1966), 376-377.

[5] W. Xue, 'On strongly right bounded finite rings', Bull. Austral. Math. Soc. 44 (1991), 353-355.

Department of Mathematics

Fujian Normal University

Fuzhou

Fujian 350007

People's Republic of China
Current address:

Department of Mathematics

University of Iowa

Iowa City IA 52242

United States of America 